\title{
AMIDST: a Java Toolbox for Scalable Probabilistic Machine Learning
}

\author{
Andrés R. Masegosa* \\ The Norwegian University \\ of Science and Technology \\ Rafael Cabañas ${ }^{\star}$ \\ Aalborg University
}

\author{
Ana M. Martínez \\ Aalborg University
}

Antonio Salmerón

University of Almería

\author{
Darío Ramos-López ${ }^{\star}$ \\ University of Almería
}

Thomas D. Nielsen

Aalborg University

\author{
Helge Langseth \\ The Norwegian University \\ of Science and Technology
}

\author{
Anders L. Madsen \\ Aalborg University \\ HUGIN EXPERT A/S
}

\begin{abstract}
The AMIDST Toolbox is a software for scalable probabilistic machine learning with a special focus on (massive) streaming data. The toolbox supports a flexible modeling language based on probabilistic graphical models with latent variables and temporal dependencies. The specified models can be learnt from large data sets using parallel or distributed implementations of Bayesian learning algorithms for either streaming or batch data. These algorithms are based on a flexible variational message passing scheme, which supports discrete and continuous variables from a wide range of probability distributions. AMIDST also leverages existing functionality and algorithms by interfacing to software tools such as Flink, Spark, MOA, Weka, R and HUGIN. AMIDST is an open source toolbox written in Java and available at http://www.amidsttoolbox.com under the Apache Software License version 2.0.
\end{abstract}

Keywords: machine learning, probabilistic graphical models, latent variable models, variational methods, scalable learning, data streams, Java 8, Apache Flink, Apache Spark

\section{Introduction}

AMIDST is a toolbox for the analysis of small and large-scale data sets using probabilistic machine learning methods based on graphical models. A probabilistic graphical model (PGM) is a framework consisting of two parts: a qualitative component in the form of a graphical model encoding conditional independence assertions about the domain being modeled; a quantitative component consisting of a collection of local probability distributions adhering to the independence properties specified in the graphical model. Collectively, the two components provide a compact representation of the joint probability distribution over the set of variables in the domain being modelled. We consider the Bayesian network (BN) framework [17, and its dynamic extension in the form of 2 -Timeslice BNs (2TBNs) 13. These frameworks provide a well-founded and principled approach for performing inference in complex (temporal) domains endowed with uncertainty.

AMIDST implements parallel and distributed algorithms for learning probabilistic graphical models with latent or unobserved variables such as Gaussian mixtures, (probabilistic) principal component analysis, hidden Markov models, (switching) linear dynamical systems, latent Dirichlet allocation, etc. More generally, the toolbox is able to learn any user-defined probabilistic (graphical) model belonging to the conjugate exponential family using novel message passing algorithms [11. Additionally, the AMIDST Toolbox has the possibility of handling large data streams by implementing (approximate) Bayesian updating. To the best of our knowledge, there is no existing software for mining data streams based on a wide range of PGMs (including latent variable

\footnotetext{
${ }^{*}$ These four authors are considered as first authors and contributed equally to this work.
} 
models); most existing tools focus on stationary data sets [14] (see Figure 22).

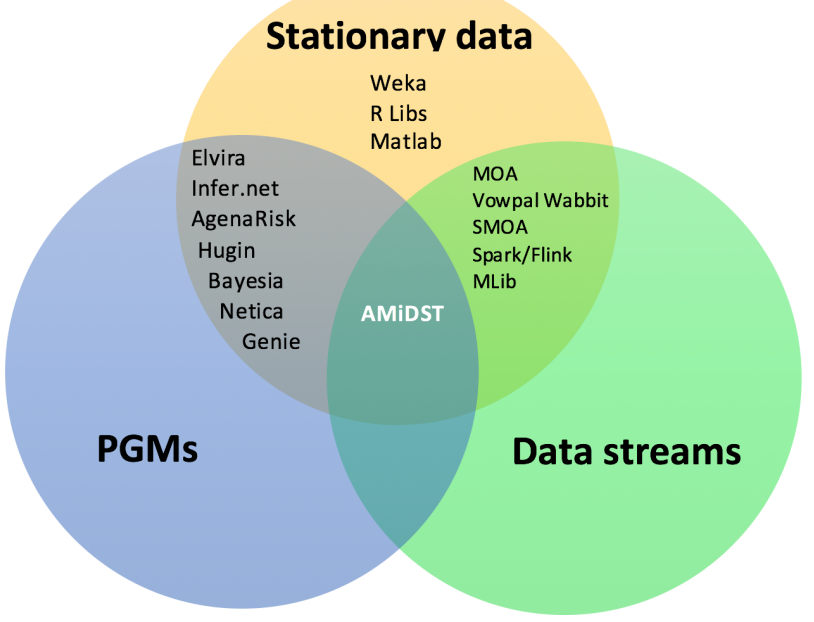

Figure 1: A taxonomy of data mining software.

In this way, AMIDST's approach to machine learning is based on the use of openbox models that can be inspected and which can incorporate prior information or knowledge about the domain, in contrast to other approaches based on blackbox models, which cannot be interpreted by the users. This is why the focus of AMIDST is not only to learn models for making predictions rather than to learn models to extract knowledge from the data 2, 1. And, as commented above, with the advantage that this toolbox is equipped with a general learning engine implementing scalable variational Bayesian inference algorithms, which allow to learn models exploiting a wide range of computational settings. The use of a Bayesian approach not only allows us to deal with model uncertainty problems but also naturally deals with data streams through the standard operation of Bayesian updating (see Section 2.3). Moreover, the probabilistic approach we pursue gives a basis to naturally deal with the presence of missing data, which is a quite ubiquitous problem in real-world applications.

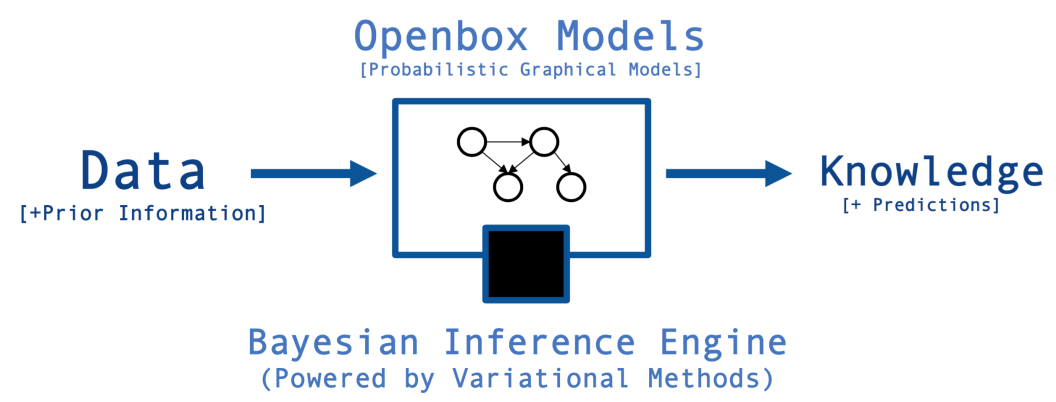

Figure 2: AMIDST's approach to machine learning.

The toolbox has been developed by the AMIDST Consortium 11, which includes both academic and industrial partners. Our software has been used in the automotive industry 20 and in the finance sector [1, 2]. The AMIDST Toolbox can be used, for instance, for classification, clustering, regression, and density estimation tasks [16]. AMIDST also offers the ability to create, learn and perform inference in (dynamic) BNs.

\footnotetext{
${ }^{1}$ http://www.amidst.eu
} 


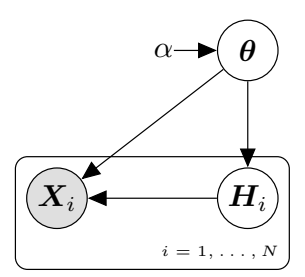

Figure 3: An example of a PGM covered by AMIDST (where $N$ is the number of samples, $\boldsymbol{X}_{i}$ a set of features, $\boldsymbol{H}_{i}$ a set of local latent variables, $\boldsymbol{\theta}$ the global parameters and $\alpha$ its

hyper-parameters).

\section{The AMIDST Toolbox features}

In what follows, we describe the main features of the AMIDST Toolbox.

\subsection{Probabilistic graphical models}

The AMIDST Toolbox supports the specification of Bayesian networks (BNs) [17, 8], which are widely used PGMs for reasoning under uncertainty. They graphically encode a set of conditional independence assumptions that can be exploited to efficiently perform a wide variety of inference tasks such as marginal belief computation, belief update, abductive inference, etc. Formally, let $\boldsymbol{X}=\left\{X_{1}, \ldots, X_{N}\right\}$ denote the set of random variables defining our domain problem. BNs can be graphically represented by a directed acyclic graph (DAG). Each node, labelled $X_{i}$ in the graph, is associated with a factor or conditional probability $p\left(X_{i} \mid \operatorname{Pa}\left(X_{i}\right)\right)$, where $\operatorname{Pa}\left(X_{i}\right) \subset \boldsymbol{X} \backslash X_{i}$ represents the so-called parent variables of $X_{i}$. Additionally, for each parent $X_{j} \in \operatorname{Pa}\left(X_{i}\right)$, the graph contains one directed edge pointing from $X_{j}$ to the child variable $X_{i}$. A BN defines a joint distribution $p(\boldsymbol{X})$ in the following form:

$$
p(\boldsymbol{X})=\prod_{i=1}^{N} p\left(X_{i} \mid \operatorname{Pa}\left(X_{i}\right)\right)
$$

Traditionally, BNs have been defined for discrete domains, where the entities of interest are modelled by discrete variables which ensures that belief updating can be performed efficiently and in closed form [17. However, this requirement imposes severe restrictions as many domains contain entities that are more appropriately modelled by variables with continuous state spaces. To deal with this problem, the AMIDST Toolbox allows the specification of conditional linear gaussian Networks (CLG) [10, 9], which are hybrid BN:2. In the CLG model, the conditional distribution of each discrete variable $X_{D} \in \boldsymbol{X}$ given its parents is a multinomial, whilst the conditional distribution of each continuous variable $Z \in \boldsymbol{X}$ with discrete parents $\boldsymbol{X}_{D} \subseteq \boldsymbol{X}$ and continuous parents $\boldsymbol{X}_{C} \subseteq \boldsymbol{X}$, is given by

$$
p\left(z \mid \boldsymbol{X}_{D}=\boldsymbol{x}_{D}, \boldsymbol{X}_{C}=\boldsymbol{x}_{C}\right)=\mathcal{N}\left(z ; \alpha\left(\boldsymbol{x}_{D}\right)+\beta\left(\boldsymbol{x}_{D}\right)^{\top} \boldsymbol{x}_{C}, \sigma\left(\boldsymbol{x}_{D}\right)\right),
$$

for all $\boldsymbol{x}_{D} \in \Omega_{\boldsymbol{X}_{D}}$ and $\boldsymbol{x}_{C} \in \Omega_{\boldsymbol{X}_{C}}$, where $\alpha$ and $\beta$ are the coefficients of a linear regression model of $Z$ given its continuous parents; this model can differ for each configuration of the discrete variables $\boldsymbol{X}_{D}$

Furthermore, latent (i.e., hidden) variables are supported. These variables cannot be observed and are included in the model to capture correlation structure. The use of latent variables allows the representation of a large range of problems with complex probabilistic dependencies. Supported PGMs generally fit to the plate structure 44 depicted in Figure 3, although more general PGMs can be considered.

The AMIDST Toolbox also allows the specification of dynamic BNs (DBNs), which are used to model domains that evolve over time by representing explicitly the temporal dynamics of the system. In the DBN framework, variables are indexed by a discrete time index $t$. For example, $X_{t}$ is the variable $X$ at time $t$. In this way, we explicitly model the state of the system at any

\footnotetext{
${ }^{2}$ The toolbox is designed to be extended to support any model belonging to the conjugate-exponential family (this class includes many of the most common distributions, such as Gaussian, mixture of Gaussian, Exponential, Dirichlet, Bernoulli, Gamma, etc.).
} 
given time. For reasoning over time, we need to model the joint probability $p\left(\boldsymbol{X}_{1: T}\right)$ which has the following natural cascade decomposition:

$$
p\left(\boldsymbol{X}_{1: T}\right)=\prod_{t=1}^{T} p\left(\boldsymbol{X}_{t} \mid \boldsymbol{X}_{1: t-1}\right)
$$

where $p\left(\boldsymbol{X}_{t} \mid \boldsymbol{X}_{1: t-1}\right)$ is equal to $p\left(\boldsymbol{X}_{1}\right)$ for $t=1$, and $\boldsymbol{X}_{1: t-1}$ is the set $\left\{X_{1}, X_{2}, \ldots X_{t-1}\right\}$.

\subsection{Scalable Bayesian inference}

The AMIDST Toolbox offers Bayesian parameter learning functionality using powerful approximate and scalable algorithms based on variational methods. AMIDST relies on the variational message passing (VMP) algorithm 21. Two versions are provided; a parallel version exploiting multi-core architectures, powered by Java 8 Streams [12]; and a novel distributed version, named d-VMP [11, for large-scale data processing on computing clusters running either Apache Flink 3 or Apache Spark. The d-VMP method has been used in the context of financial data analysis to perform efficient scalable inference in a model with more than one billion nodes (see [1] for details). Stochastic Variational Inference [7] is also available for learning.

In relation to standard inference, we also provide a parallel implementation of the importance sampling algorithm 6, 19. Versions of these methods for dynamic models are supported by means of the Factored Frontier algorithm [15]. Scalable abductive inference [18] is also supported using optimization algorithms and Monte Carlo methods.

\subsection{Batch-streaming learning}

The toolbox has the possibility to handle massive data sets and large data streams. Massive data sets can be processed in distributed computer clusters by interfacing with Apache Flink and Spark using novel scalable and distributed variational methods [11. We use a Bayesian approach for updating the model as new data arrives. Assuming $\boldsymbol{X}_{t}$ is a new batch of data at time $t, \boldsymbol{\theta}$ the parameters of the model and $\boldsymbol{H}$ the set of latent variables, the Bayesian updating is performed as follows,

$$
p\left(\boldsymbol{\theta}, \boldsymbol{H} \mid \boldsymbol{X}_{1}, \ldots, \boldsymbol{X}_{t}\right) \propto p\left(\boldsymbol{X}_{t} \mid \boldsymbol{\theta}, \boldsymbol{H}\right) p\left(\boldsymbol{\theta}, \boldsymbol{H} \mid \boldsymbol{X}_{1}, \ldots, \boldsymbol{X}_{t-1}\right) .
$$

This provides a natural setting for stream processing, and there is therefore no need to learn the model from scratch as new data arrives. When learning from streaming data AMIDST also provides an implementation of the streaming variational Bayes method [3], also based on VMP and d-VMP algorithms.

An important aspect of streaming data is that the domain being modeled is often non-stationary. That is, the distribution governing the data changes over time. This situation, which is known as concept drift [5], can be detected with AMIDST using a novel probabilistic approach [2].

\subsection{Modular design}

The AMIDST Toolbox has been designed following a modular structure, i.e., all the provided functionality is organized in different modules (Table 1 shows a brief description of all the modules in the toolbox). This modularity allows future extensions to be made independently of the core design, thereby leaving the kernel small and robust. Another added value of the modularity is that it enables a more seamlessly interaction with external software. Currently, AMIDST interfaces with MOA 5 , Weka6, and HUGIN7.

\footnotetext{
${ }^{3}$ http://flink.apache.org

${ }^{4}$ http://spark . apache.org

5 http://moa.cms.waikato.ac.nz

6 http://www.cs.waikato.ac.nz/ml/weka/

http://www.hugin.com
} 


\begin{tabular}{|l||l|}
\hline Module & Description \\
\hline \hline core & $\begin{array}{l}\text { Functionality related to (static) BNs (i.e., learning and in- } \\
\text { ference algorithms, classes handling data streams, static } \\
\\
\text { DAGs, variables, etc.). }\end{array}$ \\
\hline core-dynamic & $\begin{array}{l}\text { Analogous to the core module, but implementing the func- } \\
\text { tionality related to DBNs. }\end{array}$ \\
\hline latent-variable-models & $\begin{array}{l}\text { Contains a wide range of predefined (static and dynamic) } \\
\text { latent variable models. Learning and applying these models } \\
\text { is straight-forward, which simplifies the use of the toolbox. }\end{array}$ \\
\hline lda & $\begin{array}{l}\text { Allows text processing by means of the latent Dirichlet al- } \\
\text { location model. }\end{array}$ \\
\hline huginlink & $\begin{array}{l}\text { Implements the interaction with the external software } \\
\text { HUGIN (e.g., use of its inference engine, reading and writ- } \\
\text { ing BNs in HUGIN format, etc.) }\end{array}$ \\
\hline wekalink & $\begin{array}{l}\text { Implements the interaction with the external software } \\
\text { Weka. For example, for creating a wrapper for evaluating } \\
\text { an AMDIST classifier with Weka. }\end{array}$ \\
\hline flinklink & $\begin{array}{l}\text { Implements the integration with Apache Flink, which al- } \\
\text { lows the implemented algorithms to run in a distributed ar- } \\
\text { chitecture. }\end{array}$ \\
\hline sparklink & $\begin{array}{l}\text { Implements the integration with Apache Spark which allows } \\
\text { the implemented algorithms to run in a distributed architec- } \\
\text { ture. }\end{array}$ \\
\hline moalink & $\begin{array}{l}\text { Implements the integration with MOA library. Any model } \\
\text { developed on AMIDST can be evaluated within MOA. }\end{array}$ \\
\hline examples & $\begin{array}{l}\text { A set of code examples using the functionality provided in } \\
\text { the rest of the modules. The unit tests are integrated int } \\
\text { each module separately. }\end{array}$ \\
\hline
\end{tabular}

Table 1: Modules in AMIDST.

\section{Code-examples}

\subsection{Managing data streams in a single computer}

The DataStream class in package eu.amidst.core.datastream is an interface for dealing with data streams stored on a single computer. The whole AMIDST Toolbox is specifically designed to process the data sequentially without having to load all observations into main memory simultaneously. In this way, this class can also handle static data-sets which do not fit into main memory. A DataStream object is composed by a collection of DataInstance objects. The functionality for loading data is provided by class eu.amidst.core.io.DataStreamLoader. In particular, an example of reading data from a .arff file is given in Code Fragment 1 .

DataStream $<$ DataInstance $>$ data $=$ DataStreamLoader.open("data0.arff");

Code Fragment 1: Reading a (static) data stream from a .arff file.

When loading dynamic data streams (i.e., with temporal information), we will use the class eu. amidst.dynamic.io. DataStreamLoader instead. Now the returned object data stream will be a collection of DynamicDataInstance objects as shown in Code Fragment 2 .

DataStream $<$ DynamicDataInstance $>$ data = DynamicDataStreamLoader.open("data0.arff");

Code Fragment 2: Loading a dynamic data stream from a .arff file. 
Static and dynamic data is managed exactly in the same way as they are both handled with the interface DataStream. In Code Fragment 3 we show how to access to the attributes' names and types (lines 2 to 6), to print all the instances (line 9), and to save the data in a file (line 12). Note that, for accessing each instance in the data stream, lambda expressions provided by Java 8 are used.

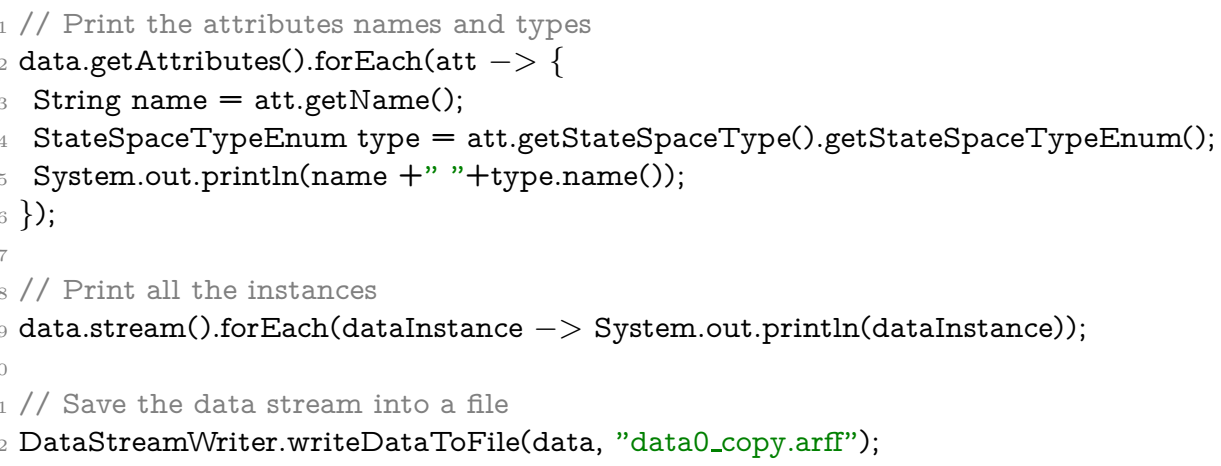

Code Fragment 3: Processing data in a multi-core environment.

In line 9 of Code Fragment 3. all the instances are sequentially processed. To do the processing in parallel, we will use the method parallelStream(int batchSize) instead of stream(). Internally, data instances are grouped into batches and all the instances in the same batch are processed with the same thread. The first lines of the output generated from the previous code are shown below.

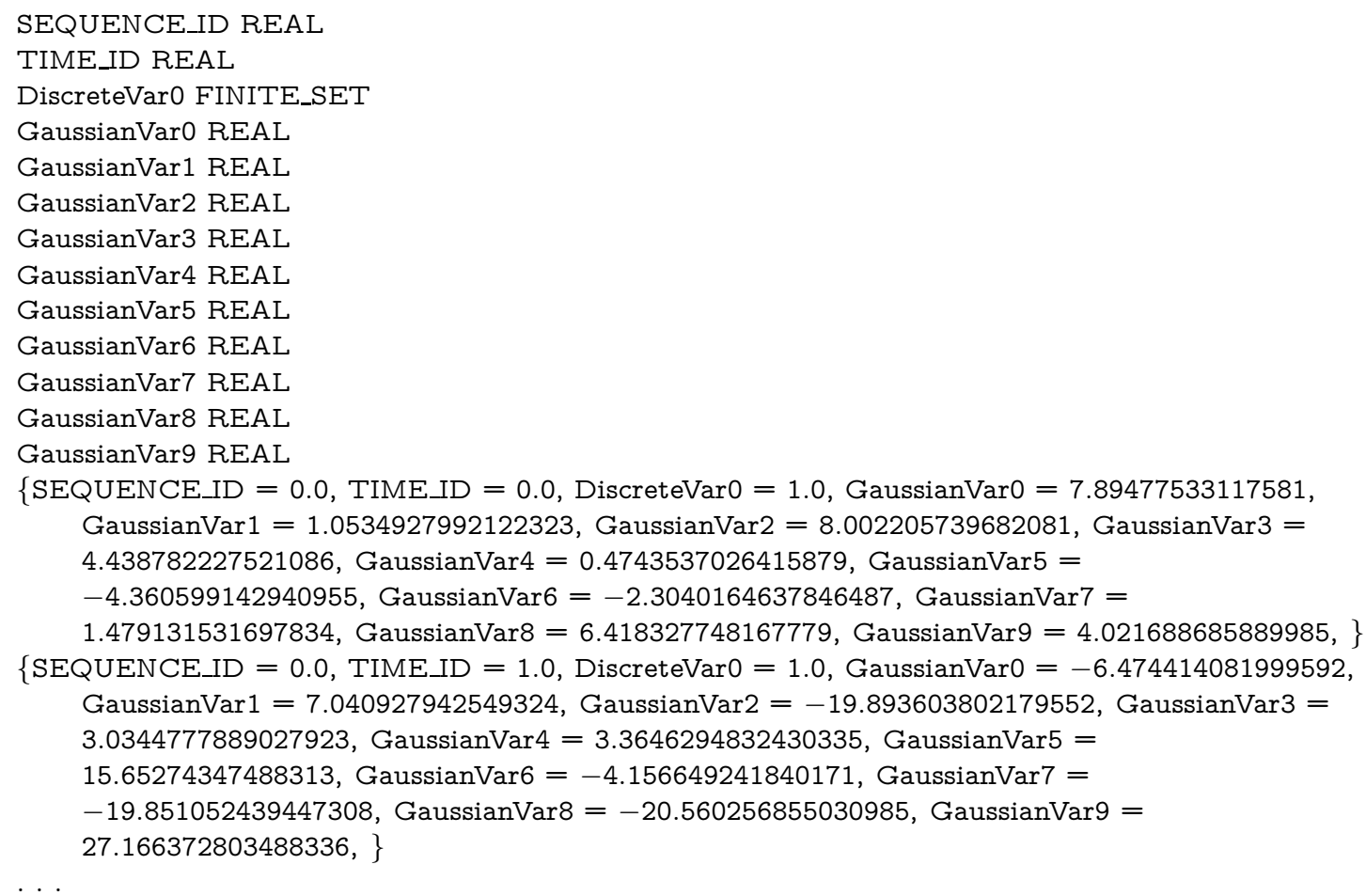

Code Fragment 4: Output generated by Code Fragment 3 . This data-set contains two special attributes specific for dynamic data: SEQUENCE_ID and TIME_ID.

\subsection{Managing data in a distributed computing cluster}

When dealing with large scale data, it might be convenient to have it stored in a distributed file system. In AMIDST, distributed data can be managed using Flink (module flinklink). Code 
Fragment 5 shows how to load data from a Hadoop Distributed File System (HDFS). In line 2, the Flink session is set up, and in line 5 the data is loaded using the static method open from class eu.amidst.flinklink. core.io.DataFlinkLoader. This method returns an object of class DataFlink<DataInstance> and takes three arguments: the Flink execution enviroment, the path to the data, and a boolean indicating whether the data should be normalized or not.

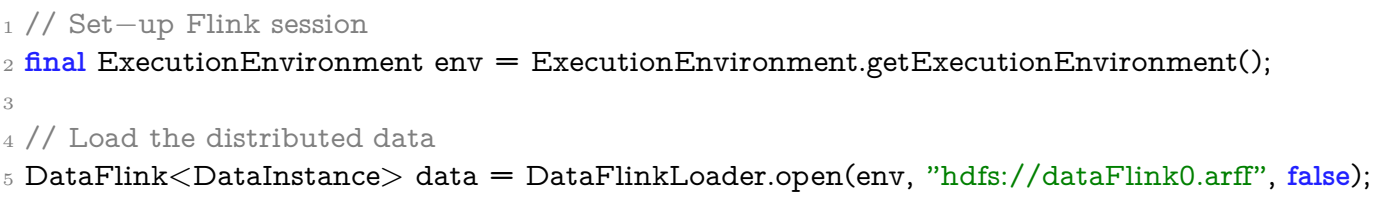

Code Fragment 5: Loading distributed data in AMIDST.

The access to the instances is slightly different compared to the multi-core case (Code Fragment 3). As shown in Code Fragment 6. in a distributed cluster, we will use the method getDataSet() from class DataFlink<DataInstance>, which returns an object of class DataSet (i.e., a class from the Flink API). The writing operation is done by invoking the static method writeDataToARFFFolder from the class eu.amidst.flinklink .core.io.DataFlinkWriter.

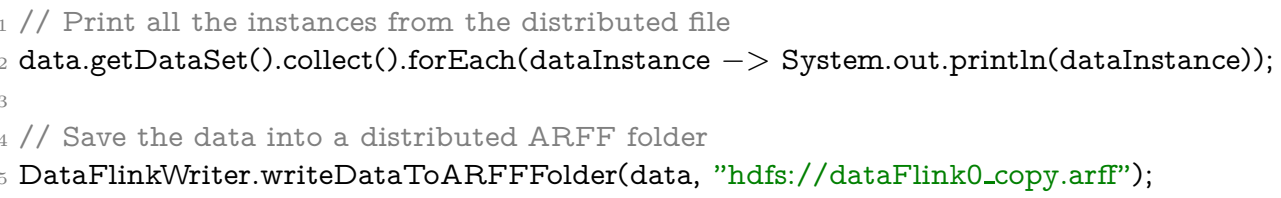

Code Fragment 6: Processing data in a distribtued environment.

\subsection{Scalable learning of probabilistic graphical models}

\subsubsection{Learning a model}

The AMIDST Toolbox contains a wide range of predefined models, most of them including latent variables (see Table 21). These models are available in the module latent-variable-models. All of them are direct instantations of the class of models supported (see Section 2.1).

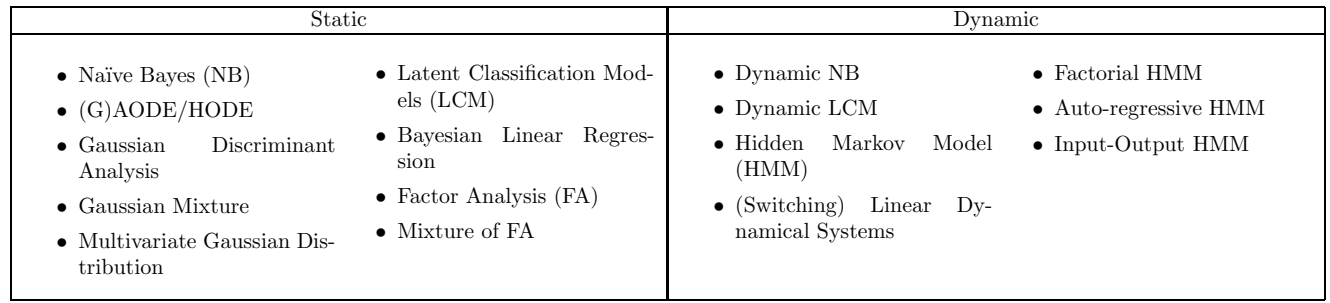

Table 2: Predefined models in AMIDST.

Learning and applying these models is straight-forward. As an example, Code Fragment 7 shows how to learn a static a Gaussian mixture model [16] with a binary latent variable and continuous observable variables. The class eu.amidst. latentvariablemodels.staticmodels.Model encapsulates all the functionality for learning the predefined static models and hence, any class implementing a particular static model should inherit it. In lines 2 to 4 , the model is built from a list of attributes. In line 6 , by invoking the method updateModel, the probability distributions are learnt from data. This method takes one argument, which can be either an object of class DataStream<DataInstance> or DataFlink<DataInstance>, which corresponds to a local multicore computer or a distributed computing cluster, respectively. Note that, in this way, the code for learning a model is independent of the processing environment (i.e., multi-core or distributed). 
Finally, an object of class BayesianNetwork is obtained and printed to the standard output (lines 7 and 8). An additional advantage of using AMIDST is that the code is flexible: if we aim to learn another predefined static model, we simply have to change the constructor used in line 3.

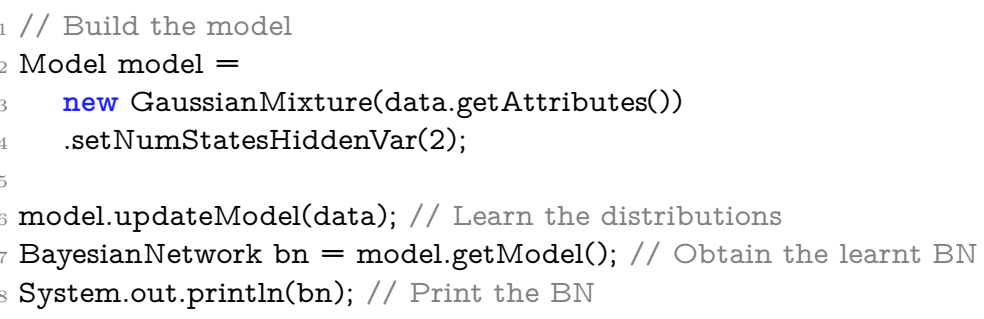

Code Fragment 7: Learning a predefined static model from data.

The output generated by Code Fragment 7 is shown below. The print-out includes the distributions associated to each variable: the discrete variables have a multinomial, while the continuous ones have normal distributions given by (2). Note that, for space restriction, the generated output has been reduced.

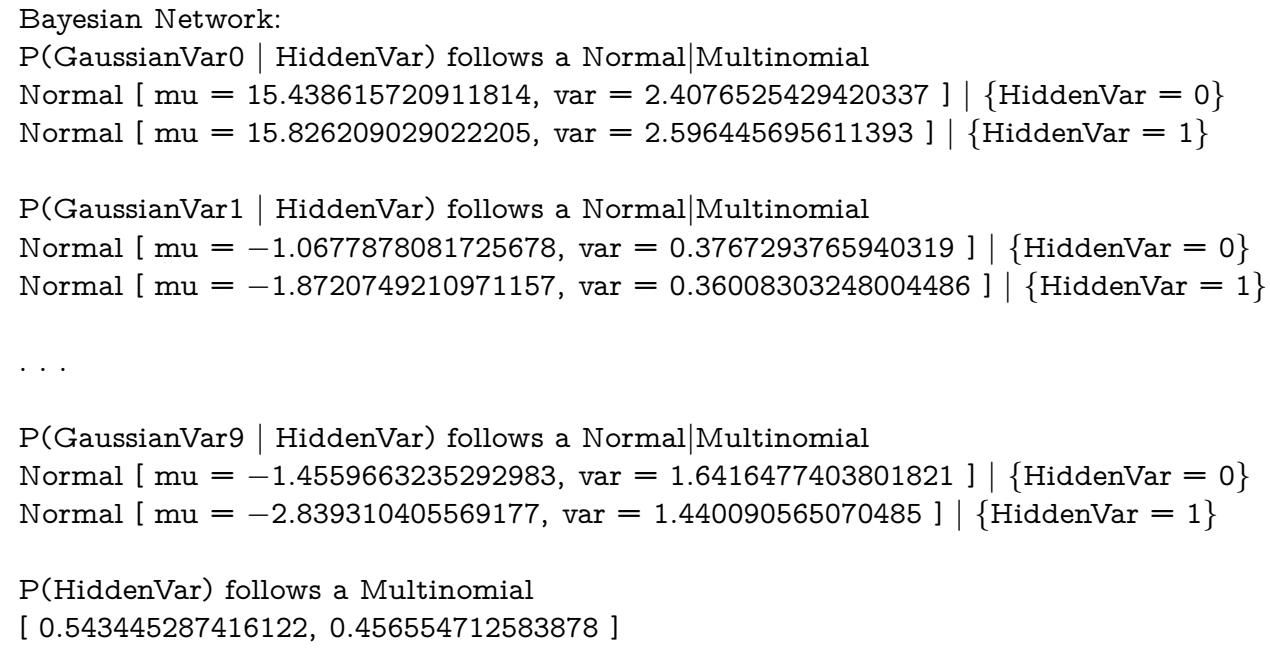

Code Fragment 8: Reduced output generated by Code Fragment 7

\subsubsection{Updating a model}

An advantage of the AMIDST Toolbox is that the model can be easily updated when new data becomes available (see Equation (3) ). This capacity is useful when analysing streaming data, as new data is constantly being generated and it could happen that the old model might not accurately represent this new data (see Code Fragment 9).

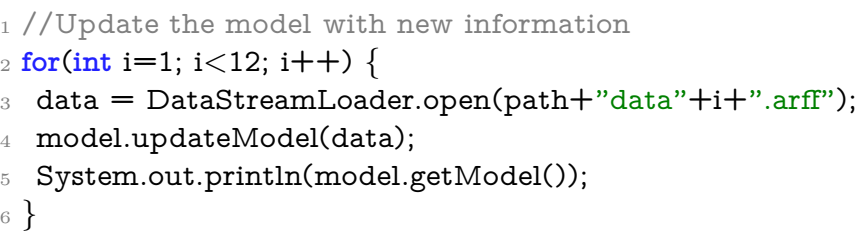

Code Fragment 9: Updating the probability distributions of a model with new data. 


\subsubsection{Learning models with temporal dependencies}

When learning a dynamic model, an object of any class that inherits from eu. amidst. latentvariablemodels. dynamicmodels. DynamicModel should be created. As an example, Code Fragment 10 shows how a Kalman Filter can be learnt from dynamic data (i.e., an object of class DataStream<DynamicDataInstance>).

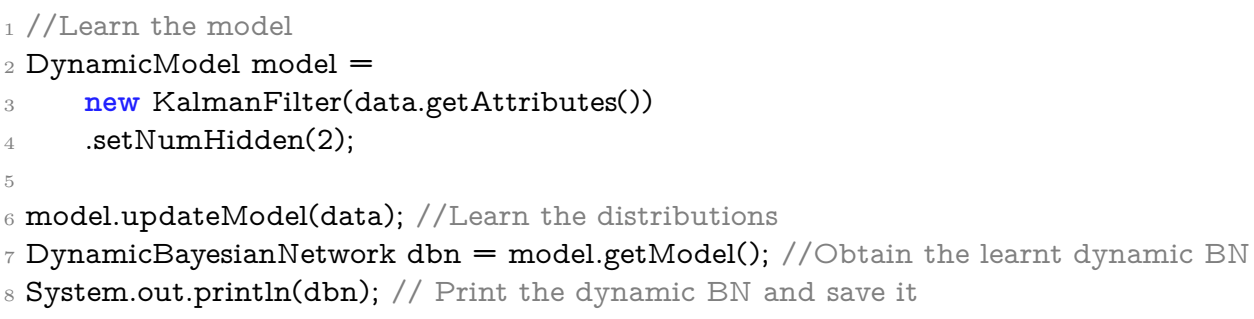

Code Fragment 10: Learning a predefined dynamic model from data.

\subsubsection{Define custom models}

If neither of the predefined models fit with the problem being modelled, a custom model can be defined. For doing that, we should create a class that inherits Model and overrides the method protected void buildDAG(). This class must contain a constructor taking as argument an object of class Attributes. Code Fragment 11 shows the definition of a Gaussian mixture model with a local latent parent of each observed variable as an example of this procedure. The most important part of the code is the method buildDAG(), where the member variable dag is initialized. An object of class DAG is basically a list of parent sets, one for each variable. Thus, to define a DAG, we should add the corresponding variables to these parent sets (lines 20 and 21).

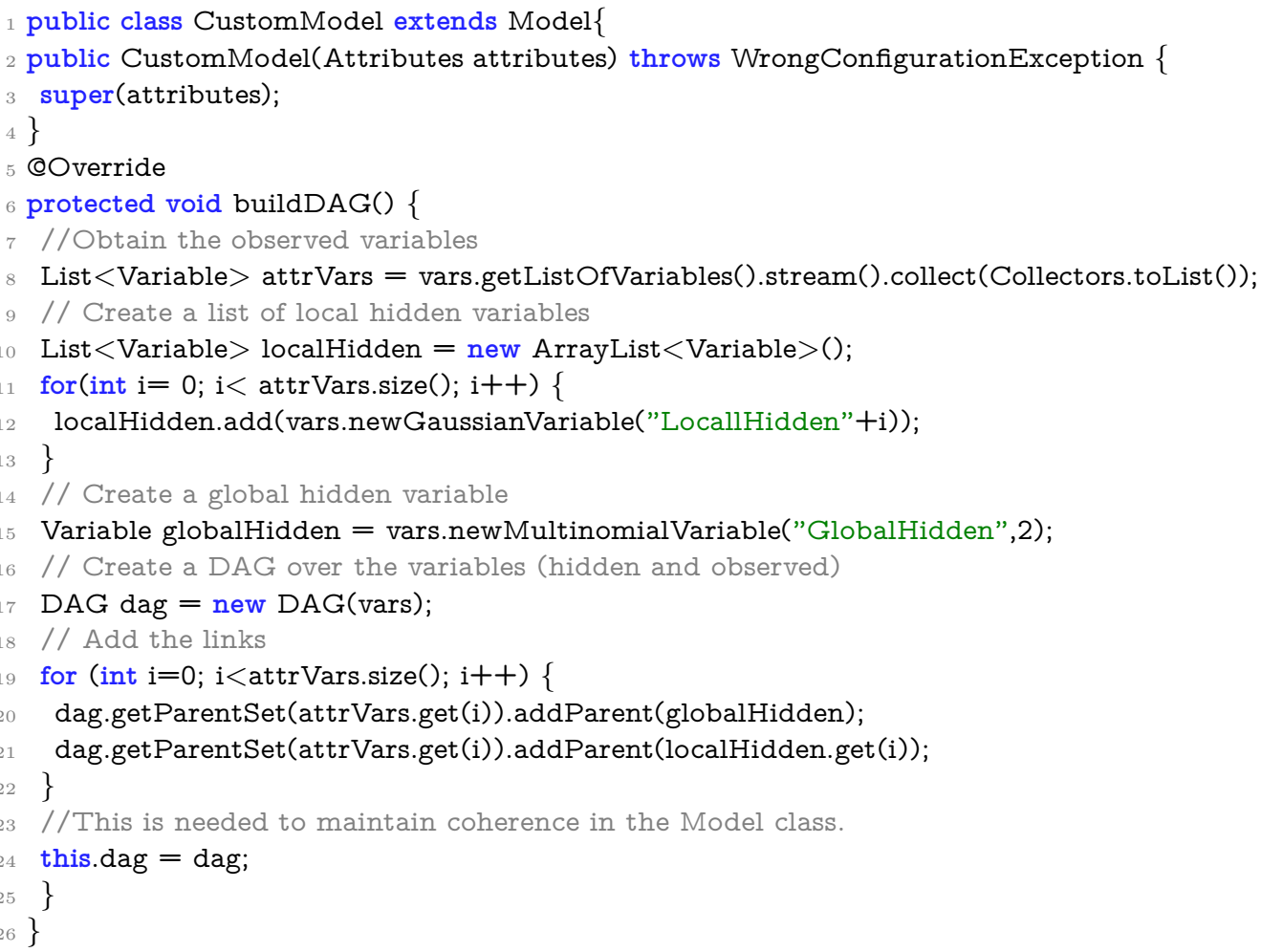

Code Fragment 11: Definition of a custom model.

To learn this custom model, we simply create an object of class CustomModel, and invoke the method updateModel as shown in Code Fragment 12. 
Model model = new CustomModel(data.getAttributes());

2 model.updateModel(data);

Code Fragment 12: Learning a custom model from data.

\subsection{Inference}

AMIDST provides inference algorithms exploiting multi-core architectures. As an example, Code Fragment 13 shows how to make inference in the Gaussian mixture learnt in Code Fragment 7. All the functionality for making inference is defined in the interface eu. amidst. core. inference. InferenceAlgorithm. Any class implementing a particular inference algorithm should implement this interface. VMP is the algorithm used in code below. This can be changed by using another constructor in line 10 (e.g., new HuginInference() or new ImportanceSampling()). By invoking the method setModel (BayesianNetwork model), we specify in which model we aim to make inference (line 11). The evidence is then set in line 12 . Finally, the inference is done by invoking the method runInference(). Afterwards, we can obtain the posterior distribution of any variable by calling the method getPosterior (Variable var).

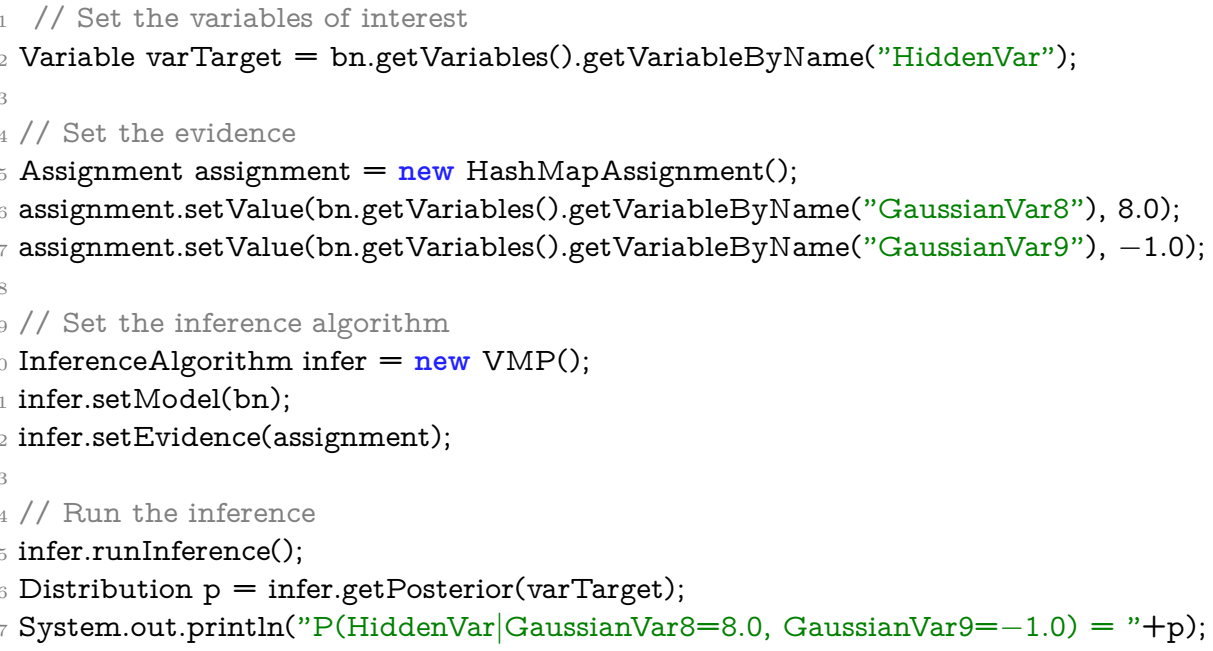

Code Fragment 13: Inference in a (static) BN using the VMP algorithm.

Analogously, the inference in a dynamic model is illustrated in Code Fragment 14. In particular, this example shows how to use the Factored Frontier algorithm with importance sampling. One difference w.r.t. the static case is that the assignment includes information about the current time (line 16). Inference is then done by invoking runInferenrece. When dealing with dynamic models, we might be interested in computing the posterior distributions at the current instant of time (line 23) or in the future (line 27).

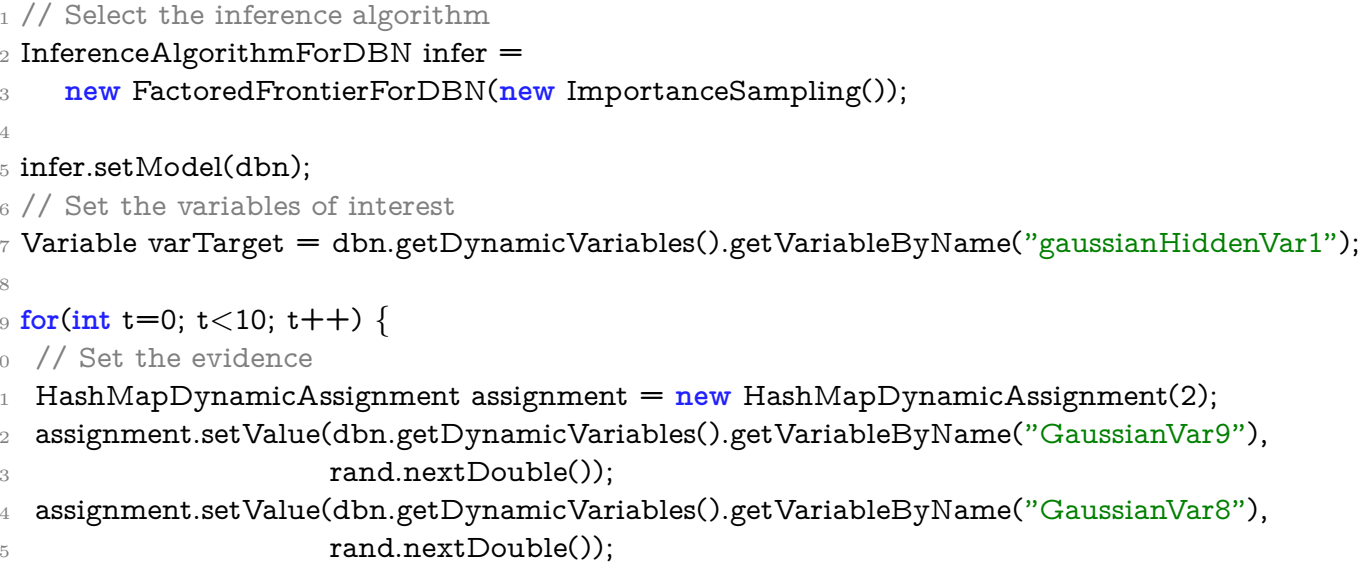




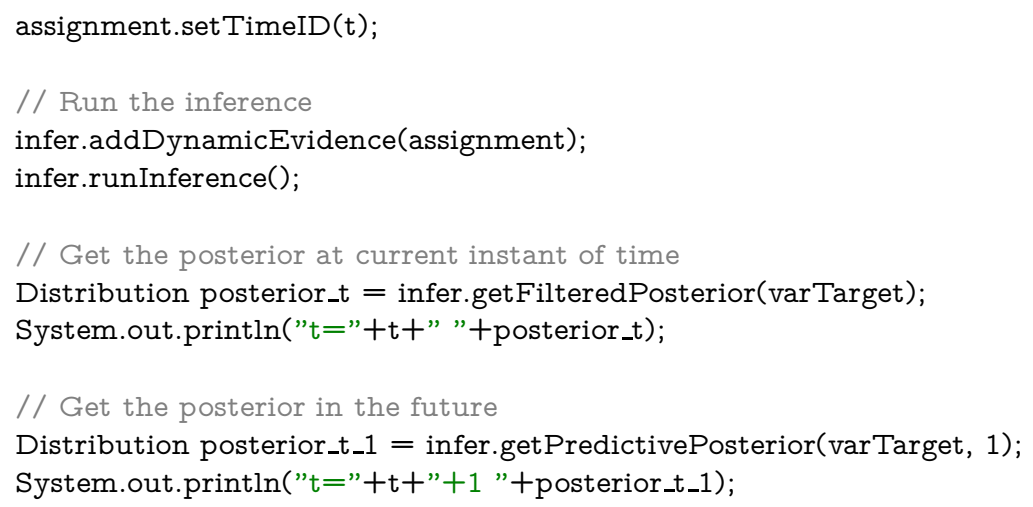

Code Fragment 14: Inference in a dynamic model.

\section{Documentation, Availability, and Further Developments}

The AMIDST Toolbox is available at http://www. amidsttoolbox.com under the Apache Software License version 2.0 and the documented source code is hosted on GitHub 8 . The website includes a tutorial explaining how to install the toolbox, and provides a large set of source code examples of the use of the toolbox API, including example implementations of well-known models (see Table 2). The code examples shown in this paper can be found in a specific repository9. This includes all the material for compiling and running the examples (scripts, java files, libraries, etc.). The toolbox is distributed using Maven 10. The use of this technology simplifies the installation making the interaction with external software transparent. In addition, public collaborations is supported and encouraged via GitHub Fork and Pull requests. The toolbox has been uploaded to the MLOSS 11 repository. Additionally, the toolbox can be acessed from $\mathrm{R}$ by means of the package ramidst 12 which provides access to some key functionalities.

\section{Acknowledgments}

This work was performed as part of the AMIDST project. AMIDST has received funding from the European Union's Seventh Framework Programme for research, technological development and demonstration under grant agreement no 619209.

\section{References}

[1] H. Borchani, A. M. Martínez, A. R. Masegosa, H. Langseth, T. D. Nielsen, A. Salmerón, A. Fernández, Anders L. Madsen, and R. Sáez. Dynamic Bayesian modeling for risk prediction in credit operations. In Proceedings of the Thirteenth Scandinavian Conference on Artificial Intelligence (SCAI 2015), pages 17-26, 2015.

[2] H. Borchani, A. M. Martínez, A. R. Masegosa, H. Langseth, T. D. Nielsen, A. Salmerón, A. Fernández, Anders L. Madsen, and R. Sáez. Modeling concept drift: A probabilistic graphical model based approach. In International Symposium on Intelligent Data Analysis, pages 72-83. Springer, 2015.

[3] T. Broderick, N. Boyd, A. Wibisono, A. C. Wilson, and M. I. Jordan. Streaming variational Bayes. In Advances in Neural Information Processing Systems, pages 1727-1735, 2013.

[4] Wray L. Buntine. Operations for learning with graphical models. JAIR, 2:159-225, 1994.

\footnotetext{
${ }^{8}$ https://github.com/amidst/toolbox

9 https://github.com/amidst/jss

10 https://maven. apache.org

11 http://mloss.org/software/

12 https://cran.r-project.org/web/packages/ramidst/
} 
[5] J. Gama, I. Žliobaitè, A. Bifet, M. Pechenizkiy, and A. Bouchachia. A survey on concept drift adaptation. ACM Computing Surveys, 46(4):44:1-44:37, 2014.

[6] J. M. Hammersley and D. C. Handscomb. Monte Carlo Methods. Methuen \& Co, London, UK, 1964.

[7] M. D. Hoffman, D. M. Blei, C. Wang, and J. Paisley. Stochastic Variational Inference. Journal of Machine Learning Research, 14:1303-1347, May 2013.

[8] F.V. Jensen and T.D. Nielsen. Bayesian networks and decision graphs. Springer Publishing Company, Incorporated, second edition, 2007.

[9] S.L. Lauritzen. Graphical Models. Oxford University Press, 1996.

[10] Steffen L. Lauritzen. Propagation of probabilities, means, and variances in mixed graphical association models. Journal of the American Statistical Association, 87(420):1098-1108, 1992.

[11] A. R. Masegosa, A. M. Martínez, H. Langseth, T. D. Nielsen, A. Salmerón, D. Ramos-López, and A. L. Madsen. d-VMP: Distributed variational message passing. In JMLR: Proceedings of the 8th International Conference on Probabilistic Graphical Models, pages 321-332, 2016.

[12] Andres R Masegosa, Ana M Martinez, and Hanen Borchani. Probabilistic graphical models on multi-core cpus using java 8. IEEE Computational Intelligence Magazine, 11(2):41-54, 2016.

[13] K. P. Murphy. Dynamic Bayesian networks: Representation, inference and learning. PhD thesis, University of California, Berkeley, 2002.

[14] K. P. Murphy. Software for graphical models: A review. International Society for Bayesian Analysis Bulletin, 14(4):13-15, 2007.

[15] K. P. Murphy and Y. Weiss. The factored frontier algorithm for approximate inference in dbns. CoRR, abs/1301.2296, 2013.

[16] Kevin P Murphy. Machine learning: a probabilistic perspective. MIT press, 2012.

[17] J. Pearl. Probabilistic Reasoning in Intelligent Systems: Networks of Plausible Inference. Morgan Kaufmann Publishers, San Mateo, CA., 1988.

[18] D. Ramos-López, A. Salmerón, R. Rumí, A. M. Martínez, T. D. Nielsen, A. R. Masegosa, H. Langseth, and A. L. Madsen. Scalable MAP inference in Bayesian networks based on a Map-Reduce approach. In Proceedings of the Eighth International Conference on Probabilistic Graphical Models, pages 415-425, 2016.

[19] A. Salmerón, D. Ramos-López, H. Borchani, A. M. Martínez, A. R. Masegosa, A. Fernández, H. Langseth, A. L. Madsen, and T. D. Nielsen. Parallel importance sampling in conditional linear Gaussian networks. In Advances in Artificial Intelligence: 16th Conference of the Spanish Association for Artificial Intelligence, pages 36-46, 2015.

[20] G. Weidl, A. L. Madsen, V. Tereshchenko, D. Kasper, and G. Breuel. Early recognition of maneuvers in highway traffic. In European Conference on Symbolic and Quantitative Approaches to Reasoning and Uncertainty, pages 529-540. Springer, 2015.

[21] J. M. Winn and C. M. Bishop. Variational message passing. Journal of Machine Learning Research, 6:661-694, 2005. 